\title{
On the modified Randic index of trees, unicyclic graphs and bicyclic graphs
}

\author{
Jianping Li and Bo Zhou
}




\title{
ON THE MODIFIED RANDIĆ INDEX OF TREES, UNICYCLIC GRAPHS AND BICYCLIC GRAPHS
}

\author{
JIANPING LI AND BO ZHOU
}

Received 10 January, 2012

Abstract. The modified Randić index of a graph $G$ is a graph invariant closely related to the classical Randić index, defined as

$$
R^{\prime}(G)=\sum_{u v \in E(G)} \frac{1}{\max \left\{d_{G}(u), d_{G}(v)\right\}},
$$

where $E(G)$ is the edge set of $G, d_{G}(u)$ is the degree of vertex $u$ in $G$. We obtain best possible lower bounds for the modified Randić index of trees, unicyclic graphs and bicyclic graphs respectively when the number of vertices and matching number are fixed.

2000 Mathematics Subject Classification: 05C35; 05C90

Keywords: Randić index, modified Randić index, tree, unicyclic graph, bicyclic graph, matching number

\section{INTRODUCTION}

Let $G$ be a simple graph with vertex set $V(G)$ and edge set $E(G)$. For $u \in V(G)$, $N_{G}(u)$ denotes the set of neighbors of $u$ in $G$ and the degree of $u$ is $d_{G}(u)=$ $\left|N_{G}(u)\right|$. The Randić (connectivity) index of $G$ is defined as [12]

$$
R(G)=\sum_{u v \in E(G)} \frac{1}{\sqrt{d_{G}(u) d_{G}(v)}} .
$$

The Randić index is one of the most successful molecular descriptors in structureproperty and structure-activity relationships studies [9, 12]. Its mathematical properties as well as those of its generalizations have been studied extensively as summarized in the books $[7,10]$.

Various variants of Randić index have been proposed in the literature, see, e.g., $[2,4,11,13,15]$. To investigate the relationship between Randić index and radius of a (connected) graph, Dvořák et al. [6] proposed a modified version, which we call the

The second author (corresponding author) was supported by the Guangdong Provincial Natural Science Foundation of China (Grant No. S2011010005539). 
modified Randić index, defined as

$$
R^{\prime}(G)=\sum_{u v \in E(G)} \frac{1}{\max \left\{d_{G}(u), d_{G}(v)\right\}} .
$$

Some basic mathematical properties of the modified Randić index have been established in $[1,3,6]$. Recall that a connected graph on $n$ vertices is known as a tree, a unicyclic graph and a bicyclic graph if it possesses $n-1, n$ and $n+1$ edges, respectively. Andova et al. [1] obtained best possible lower and upper bounds for the modified Randić index of $n$-vertex trees and unicyclic graphs, respectively, and determined the corresponding extremal graphs.

In this paper, we obtain best possible lower bounds for the modified Randic index of trees, unicyclic graphs and bicyclic graphs respectively when the number of vertices and matching number are fixed. We also obtain best possible lower and upper bounds for the modified Randić index of $n$-vertex bicyclic graphs, and determine the corresponding extremal graphs.

\section{Preliminaries}

A matching $M$ of the graph $G$ is a subset of $E(G)$ such that no two edges in $M$ share a common vertex. The matching number of $G$ is the maximum number of edges of a matching in $G$. An $m$-matching is a matching of size $m$.

If $M$ is a matching of a graph $G$ and vertex $v \in V(G)$ is incident with an edge of $M$, then $v$ is said to be $M$-saturated, and if every vertex of $G$ is $M$-saturated, then $M$ is a perfect matching.

For a graph $G$ with $u, v \in V(G), G-u$ denotes the graph formed from $G$ by deleting vertex $u$ (and its incident edges), $G+u v$ denotes the graph formed from $G$ by adding the edge $u v$ if $u v \notin E(G)$, and $G-u v$ denotes the graph formed from $G$ by deleting the edge $u v$ if $u v \in E(G)$.

First we give some lemmas that will be used.

Lemma 1. [1] Let $G$ be a connected graph on at least 3 vertices. Let $u$ be a pendent vertex of $G$ and $v$ the unique neighbor of $u$. Let $a=d_{G}(v)$ and let $l$ be the number of neighbors of $v$ whose degree is at least a in $G$. Then

$$
R^{\prime}(G)-R^{\prime}(G-u)=\frac{l}{a(a-1)}
$$

Lemma 2. Let $G$ be a connected graph on at least 5 vertices. Let $u$ be a pendent vertex of $G$ and $v$ the unique neighbor of $u$. Suppose that $d_{G}(v)=2$. Let $w$ be the neighbor of $v$ different from $u$. Let $a=d_{G}(w)$ and let $l$ be the number of neighbors of $w$ whose degree is at least $a$ in $G$. Then

$$
R^{\prime}(G)-R^{\prime}(G-u-v)= \begin{cases}1 & \text { if } a=2 \\ \frac{1}{2}+\frac{l}{a(a-1)} & \text { if } a \geq 3\end{cases}
$$


Proof. Obviously, $a \geq 2$. Suppose first that $a=2$. Let $w_{1}$ be the neighbor of $w$ different from $v$. Obviously, $d_{G-u-v}\left(w_{1}\right)=d_{G}\left(w_{1}\right) \geq 2$. Note that only edges $u v$ and $v w$ affect the difference $R^{\prime}(G)-R^{\prime}(G-u-v)$. Thus $R^{\prime}(G)-R^{\prime}(G-u-v)=$ $\frac{1}{2}+\frac{1}{2}=1$. Now suppose that $a \geq 3$. By Lemma $1, R^{\prime}(G)-R^{\prime}(G-u)=\frac{1}{2}$ and $R^{\prime}(G-u)-R^{\prime}(G-u-v)=\frac{l}{a(a-1)}$. The result follows easily.

Let $P_{n}$ be the path on $n$ vertices. To attaching a path $P$ at a vertex $u$ of a graph $G$ mean that we add an edge between $u$ and an end vertex of $P$.

Lemma 3. Let $G$ be a connected graph with at least two vertices and let $u \in V(G)$. Let $G_{1}$ be the graph obtained from $G$ by attaching two paths $P_{a}$ and $P_{b}$ to $u, G_{2}$ the graph obtained from $G$ by attaching $a$ path $P_{a+b}$ to $u$, where $a \geq b \geq 1$. Then $R^{\prime}\left(G_{1}\right)<R^{\prime}\left(G_{2}\right)$.

Proof. Let $d=d_{G}(u)$. Then $d_{G_{1}}(u)=d+2$ and $d_{G_{2}}(u)=d+1$. Thus

$$
\begin{aligned}
R^{\prime}\left(G_{2}\right)-R^{\prime}\left(G_{1}\right)= & \sum_{v \in N_{G}(u)}\left(\frac{1}{\max \left\{d_{G}(v), d+1\right\}}-\frac{1}{\max \left\{d_{G}(v), d+2\right\}}\right) \\
& +\frac{1}{2}+\frac{1}{d+1}-\frac{2}{d+2} \\
\geq & \frac{1}{2}+\frac{1}{d+1}-\frac{2}{d+2} \\
> & 0,
\end{aligned}
$$

implying the result.

Lemma 4. Let $G$ be a connected graph with $u \in V(G)$, where $d_{G}(u) \geq 2$. Let $H$ be the graph obtained from $G$ by attaching a path $P_{a}$ to $u$. Let $u_{1}$ be a neighbor of $u$ in $G$, and $u_{0}$ the pendent vertex of the attached path in $H$. Let $H^{\prime}=H-u u_{1}+$ $u_{0} u_{1}$. Then $R^{\prime}\left(H^{\prime}\right)>R^{\prime}(H)$.

Proof. Let $d=d_{G}(u)$. Then $d_{H}(u)=d+1$ and $d_{H^{\prime}}(u)=d$. Note that $d_{H}\left(u_{0}\right)=$ $1, d_{H^{\prime}}\left(u_{0}\right)=2$, and the vertices of $V(H) \backslash\left\{u, u_{0}\right\}$ have the same degree in $H^{\prime}$ and $H$. Then

$$
\begin{aligned}
R^{\prime}\left(H^{\prime}\right)-R^{\prime}(H)= & \sum_{v \in N_{G}(u) \backslash\left\{u_{1}\right\}}\left(\frac{1}{\max \left\{d_{G}(v), d\right\}}-\frac{1}{\max \left\{d_{G}(v), d+1\right\}}\right) \\
& +\frac{1}{d}-\frac{1}{d+1} \\
& +\frac{1}{\max \left\{d_{G}\left(u_{1}\right), 2\right\}}-\frac{1}{\max \left\{d_{G}\left(u_{1}\right), d+1\right\}} \\
\geq & \frac{1}{d}-\frac{1}{d+1}>0,
\end{aligned}
$$

implying the result. 
A vertex $u \in V(G)$ is called a vertex of local maximal degree if $d_{G}(v)<d_{G}(u)$ for any $v \in N_{G}(u)$.

\section{MOdiFIED RANDić INDEX OF TREES}

Let $S_{n}$ be the star on $n$ vertices. For positive integers $n$ and $m$ with $n \geq 2 m$, let $\mathbb{T}_{n, m}$ be the set of trees with $n$ vertices and matching number $m$, and $T_{n, m}$ the graph obtained from the star $S_{n-2 m+2}$ by attaching $m-1$ paths on two vertices to its center.

Lemma 5. [8] Let $G$ be a $n$-vertex tree with a perfect matching, where $n \geq 3$. Then $G$ has at least two pendent vertices such that they are adjacent to vertices of degree 2.

Lemma 6. [8] Let $G$ be an n-vertex tree with matching number $m$, where $n>$ $2 m$. Then there is an m-matching $M$ and a pendent vertex $v$ such that $v$ is not $M$ saturated.

Theorem 1. Let $G \in \mathbb{T}_{n, m}$, where $n \geq 2 m$. Then $R^{\prime}(G) \geq \frac{m+1}{2}$ with equality if and only if $G \cong T_{n, m}$.

Proof. We prove the result by induction on $n$ (for fixed $m$ ).

First suppose that $n=2 m$. We prove the result by induction on $m$. If $m=1,2$, then the result follows trivially. If $m=3$, then $G \cong P_{6}$ or $T_{6,3}$, and thus the result follows since $R^{\prime}\left(T_{6,3}\right)=2=\frac{3+1}{2}<R^{\prime}\left(P_{6}\right)=\frac{5}{2}$.

Suppose that $m \geq 4$, and the theorem holds for $m-1$. Let $G \in \mathbb{T}_{2 m, m}$. Let $M$ be a perfect matching of $G$. By Lemma $5, G$ has a pendent vertex $u_{1}$ adjacent to a vertex $u_{2}$ of degree 2 . Let $u_{3}$ be the neighbor of $u_{2}$ different from $u_{1}$. Let $a=d_{G}\left(u_{3}\right)$ and $l$ be the number of neighbors of $u_{3}$ whose degree is at least $a$ in $G$. Obviously, $u_{1} u_{2} \in M$. Let $G^{\prime}=G-u_{1}-u_{2}$. Then $G^{\prime} \in \mathbb{T}_{2 m-2, m-1}$ and $M \backslash\left\{u_{1} u_{2}\right\}$ is a perfect matching of $G^{\prime}$. By Lemma 2 and the induction hypothesis, $R^{\prime}(G)=R^{\prime}\left(G^{\prime}\right)+1 \geq \frac{(m-1)+1}{2}+1>\frac{m+1}{2}$ if $a=2$, and $R^{\prime}(G)=R^{\prime}\left(G^{\prime}\right)+\frac{1}{2}+$ $\frac{l}{a(a-1)} \geq \frac{(m-1)+1}{2}+\frac{1}{2}+\frac{l}{a(a-1)}=\frac{m+1}{2}+\frac{l}{a(a-1)} \geq \frac{m+1}{2}$ with equalities if and only if $G^{\prime} \cong T_{2 m-2, m-1}$ and $l=0$, i.e., $G^{\prime} \cong T_{2 m-2, m-1}$ and $u_{3}$ is a vertex of local maximal degree in $G$ if $a \geq 3$. Thus $R^{\prime}(G) \geq \frac{m+1}{2}$ with equality if and only if $G \cong T_{2 m, m}$.

Suppose that $n>2 m$ and the result holds for $n-1$. Let $G \in \mathbb{T}_{n, m}$. By Lemma 6, $G$ has an $m$-matching $M$ and a pendent $u$ such that $M$ does not saturate $u$. Then $G-u \in \mathbb{T}_{n-1, m}$. Let $v$ be the unique neighbor of $u$ in $G$. Let $a=d_{G}(v)$ and let $l$ be the number of neighbors of $v$ whose degree is at least $a$ in $G$. By Lemma 1 and the induction hypothesis,

$$
R^{\prime}(G)=R^{\prime}(G-u)+\frac{l}{a(a-1)} \geq \frac{m+1}{2}+\frac{l}{a(a-1)} \geq \frac{m+1}{2}
$$



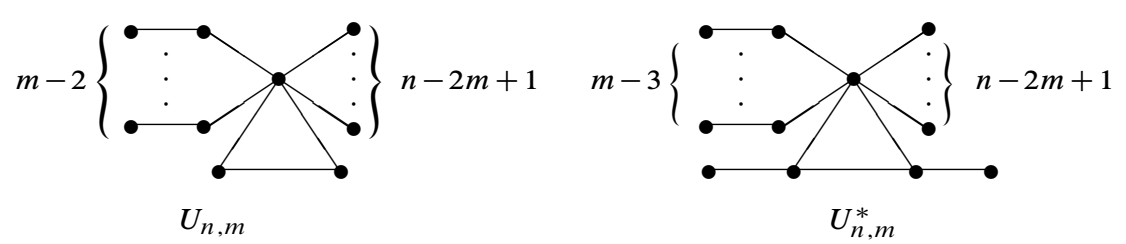

FIGURE 1. The graphs $U_{n, m}$ and $U_{n, m}^{*}$.

with equalities if and only if $G-u \cong T_{n-1, m}$ and $l=0$, i.e., $G-u \cong T_{n-1, m}$ and $v$ is a vertex of local maximal degree in $G$. Thus $R^{\prime}(G) \geq \frac{m+1}{2}$ with equality if and only if $G \cong T_{n, m}$.

Corollary 1. [1] Let $G$ be a tree on $n \geq 2$ vertices. Then $R^{\prime}(G) \geq 1$ with equality if and only if $G \cong S_{n}$.

Proof. Let $m$ be the matching number of $G$. By Theorem $1, R^{\prime}(G) \geq \frac{m+1}{2} \geq 1$ with equalities if and only if $G \cong T_{n, 1} \cong S_{n}$.

By Lemma 3, we have

Corollary 2. [1] Let $G$ be a tree on at least 2 vertices. Then $R^{\prime}(G) \leq \frac{n-1}{2}$ with equality if and only if $G \cong P_{n}$.

\section{MODIFIED RANDIĆ INDEX OF UNICYCLIC GRAPHS}

Let $C_{n}$ be a cycle on $n$ vertices. For integers $n$ and $m$ with $n \geq 2 m$ and $m \geq 2$, let $\mathbb{U}_{n, m}$ be the set of unicyclic graph with $n$ vertices and matching number $m$, and $U_{n, m}$ the graph obtained from $C_{3}$ by attaching to a vertex $n-2 m+1$ pendent vertices and $m-2$ paths on two vertices. For $n \geq 2 m$ and $m \geq 3$, let $U_{n, m}^{*}$ be the graph obtained from $C_{3}$ by attaching to a vertex $n-2 m+1$ pendent vertices and $m-3$ paths on two vertices and to the other two vertices a pendent vertex each. See Figure 1 for them.

Lemma 7. [14] Let $G \in \mathbb{U}_{n, m}$ and $G \not C_{n}$, where $n>2 m$. Then there is an $m$-matching $M$ and a pendent vertex $v$ such that $M$ does not saturate $v$.

Theorem 2. Let $G \in \mathbb{U}_{n, m}$, where $n \geq 2 m$. Then $R^{\prime}(G) \geq \frac{m+1}{2}$ with equality if and only if $G \cong U_{n, m}$ or $U_{n, m}^{*}$.

Proof. We prove the result by induction on $n$ (for fixed $m$ ).

First suppose that $n=2 m$. We prove the result by induction on $m$. 

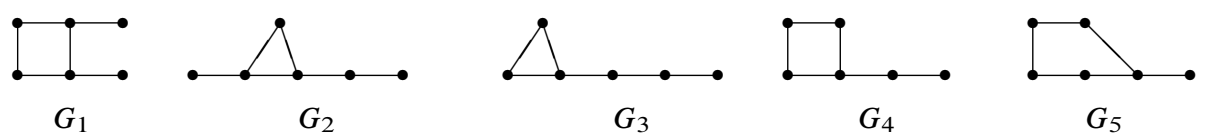

FIGURE 2. Graphs in $\mathbb{U}_{6,3} \backslash\left\{C_{6}, U_{6,3}, U_{6,3}^{*}\right\}$.

If $m=2$, then $G \cong U_{4,2}$ or $C_{4}$, and thus the result follows since $R^{\prime}\left(U_{4,2}\right)=\frac{3}{2}<$ $R^{\prime}\left(C_{4}\right)=2$. If $m=3$, then $G \cong C_{6}, U_{6,3}, U_{6,3}^{*}$ or $G_{i}$ for $i=1,2, \ldots, 5$, see Figure 2 , and thus the result follows since

$$
R^{\prime}(G)= \begin{cases}\frac{13}{6} & \text { if } G \cong G_{i}, i=1,2 \\ 3 & \text { if } G \cong C_{6} \\ \frac{5}{2} & \text { if } G \cong G_{i}, i=3,4,5 \\ 2 & \text { if } G \cong U_{6,3} \text { or } U_{6,3}^{*}\end{cases}
$$

Suppose that $m \geq 4$ and the result holds for graphs in $\mathbb{U}_{2 m-2, m-1}$. Let $G \in \mathbb{U}_{2 m, m}$. If $G \cong C_{2 m}$, then $R^{\prime}(G)=m>\frac{m+1}{2}$. Suppose that $G \nsubseteq C_{2 m}$. Let $M$ be a perfect matching of $G$. There are two cases.

Case 1. $G$ has a pendent vertex $u$ whose unique neighbor $v$ has degree two. Obviously, $u v \in M$. Let $G_{1}=G-u-v$. Then $G_{1} \in \mathbb{U}_{2 m-2, m-1}$ and $M \backslash\{u v\}$ is a perfect matching of $G_{1}$. Let $w$ be the neighbor of $v$ different from $u$ in $G$. Let $a=d_{G}(w)$ and let $l$ be the number of neighbors of $w$ whose degree is at least $a$ in $G$. By Lemma 2 and the induction hypothesis, $R^{\prime}(G)=R^{\prime}\left(G_{1}\right)+1 \geq \frac{(m-1)+1}{2}+1>\frac{m+1}{2}$ if $a=2$, and $R^{\prime}(G)=R^{\prime}\left(G_{1}\right)+\frac{1}{2}+\frac{l}{a(a-1)} \geq \frac{(m-1)+1}{2}+\frac{1}{2}=\frac{m+1}{2}$ with equality if and only if $G_{1}=U_{2 m-2, m-1}$ or $U_{2 m-2, m-1}^{*}$ and $l=0$, i.e., $G_{1}=U_{2 m-2, m-1}$ or $U_{2 m-2, m-1}^{*}$ and $w$ is a vertex of local maximal degree in $G$ if $a \geq 3$. Thus $R^{\prime}(G) \geq \frac{m+1}{2}$ with equality if and only if $G \cong U_{2 m, m}$ or $U_{2 m, m}^{*}$.

Case 2. No neighbor of a pendent vertex has degree two in $G$. Let $C=u_{1} u_{2} \ldots u_{p} u_{1}$ be the unique cycle of $G$. Since $G$ has a perfect matching, the graph obtained from $G$ by deleting vertices of $C$ consists of isolated vertices. Thus $G$ is obtainable from the cycle $C$ by attaching some pendent vertices and the maximal degree of $G$ is three.

If all vertices on $C$ have degree three, then $R^{\prime}(G)=\frac{2 m}{3}>\frac{m+1}{2}$. Suppose that there is at least one vertex of degree two on $C$. Since $G \not C_{2 m}$, there are adjacent vertices on $C$, one of degree two and the other of degree three. Assume that $d_{G}\left(u_{2}\right)=3$ and $d_{G}\left(u_{3}\right)=2$. Let $w_{2}$ be the pendent neighbor of $u_{2}$ in $G$. Then $u_{2} w_{2} \in M$. Since $u_{3} u_{4} \in M$, we have $d_{G}\left(u_{4}\right)=2$. Let $G_{2}=G-u_{2}-w_{2}+u_{1} u_{3}$. Then $G_{2} \in \mathbb{U}_{2 m-2, m-1}$ and $M \backslash\left\{u_{2} w_{2}\right\}$ is a perfect matching of $G_{2}$. If $d_{G}\left(u_{1}\right)=3$, then by the induction hypothesis,

$$
R^{\prime}(G)=R^{\prime}\left(G_{2}\right)+\frac{2}{3} \geq \frac{(m-1)+1}{2}+\frac{2}{3}>\frac{m+1}{2} .
$$


If $d_{G}\left(u_{1}\right)=2$, then $|V(C)| \geq 5$, and thus $G_{2} \not \equiv U_{2 m-2, m-1}, U_{2 m-2, m-1}^{*}$, implying that

$$
R^{\prime}(G)=R^{\prime}\left(G_{2}\right)+\frac{1}{2}>\frac{(m-1)+1}{2}+\frac{1}{2}=\frac{m+1}{2} .
$$

Thus $R^{\prime}(G)>\frac{m+1}{2}$.

By combining Cases 1 and 2, the result follows for $n=2 m$.

Suppose that $n>2 m$ and the result holds for $n-1$. Let $G \in \mathbb{U}_{n, m}$. If $G \cong C_{n}$, then $n=2 m+1$, and thus $R^{\prime}(G)=\frac{2 m+1}{2}>\frac{m+1}{2}$. Suppose that $G \nsubseteq C_{n}$. By Lemma 7, $G$ has an $m$-matching $M$ and a pendent $u$ such that $M$ does not saturate $u$. Let $G_{3}=G-u$. Then $G_{3} \in \mathbb{U}_{n-1, m}$. Let $v$ be the unique neighbor of $u$ in $G$. Let $a=d_{G}(v)$ and let $l$ be the number of neighbors of $v$ whose degree is at least $a$ in $G$. By Lemma 1 and the induction hypothesis,

$$
R^{\prime}(G)=R^{\prime}\left(G_{3}\right)+\frac{l}{a(a-1)} \geq \frac{m+1}{2}+\frac{l}{a(a-1)} \geq \frac{m+1}{2}
$$

with equalities if and only if $G_{3} \cong U_{n-1, m}$ or $U_{n-1, m}^{*}$ and $l=0$, i.e., $G_{3} \cong U_{n-1, m}$ or $U_{n-1, m}^{*}$, and $v$ is a vertex of local maximal degree in $G$. Thus $R^{\prime}(G) \geq \frac{m+1}{2}$ with equality if and only if $G \cong U_{n, m}$ or $U_{n, m}^{*}$.

Corollary 3. [1] Let $G$ be a unicyclic graph $G$ on $n \geq 3$ vertices. Then $R^{\prime}(G) \geq \frac{3}{2}$ with equality if and only if $G \cong C_{3}$ or $U_{n, 2}$.

Proof. Let $m$ be the matching number of $G$. If $m=1$, then $G \cong C_{3}$ and $R^{\prime}(G)=$ $\frac{3}{2}$. If $m \geq 2$, then by Theorem $2, R^{\prime}(G) \geq \frac{m+1}{2} \geq \frac{3}{2}$ with equalities if and only if $G \cong U_{n, 2}$.

By Lemmas 3 and 4, we have

Corollary 4. [1] Let $G$ be a unicyclic graph on at least 3 vertices. Then $R^{\prime}(G) \leq$ $\frac{n}{2}$ with equality if and only if $G \cong C_{n}$.

\section{MODIFIED RANDIĆ INDEX OF BICYCLIC GRAPHS}

In this section, we use techniques developed from [5].

For integers $n$ and $m$ with $n \geq 2 m$ and $m \geq 2$, let $\mathbb{B}_{n, m}$ be the set of bicyclic graphs with $n$ vertices and matching number $m$. Let $B_{n, 3}^{1}$ be the graph obtained by identifying a vertex of two triangles, and attaching to this common vertex of the two triangles $n-5$ pendent vertices. Let $B_{n, 3}^{2}$ be the graph obtained from the unique bicyclic graph on four vertices by attaching to a vertex of degree three $n-5$ pendent vertices and to a vertex of degree two a pendent vertex. For $n \geq 2 m$ and $m \geq 4$, let $B_{n, m}$ be the graph obtained from the unique bicyclic graph on four vertices by attaching to a vertex of degree three $n-2 m+1$ pendent vertices and $m-4$ paths on two vertices to the three other vertices a pendent vertex each, see Figure 3. 


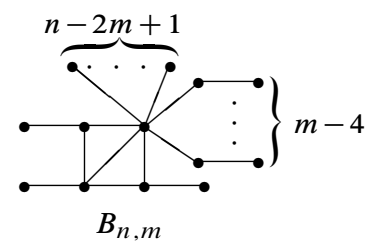

FIGURE 3. The graph $B_{n, m}$.

Lemma 8. Let $G$ be a connected graph with $u v \in E(G)$, where $d_{G}(u), d_{G}(v) \geq 2$, and $u$ and $v$ have no common neighbor in $G$. Let $G_{1}$ be the graph obtained from $G$ by deleting the edge $u v$, identifying $u$ and $v$, which is denoted by $w$, and adding $a$ vertex $w_{1}$ and the edge $w_{1} w$. Then $R^{\prime}(G)>R^{\prime}\left(G_{1}\right)$.

Proof. Note that $d_{G_{1}}(w)=d_{G}(u)+d_{G}(v)-1$, and the vertices of $V(G) \backslash\{u, v\}$ have the same degree in $G_{1}$ and $G$. Obviously, $d_{G_{1}}(w)>\max \left\{d_{G}(u), d_{G}(v)\right\}$ since $d_{G}(u), d_{G}(v) \geq 2$, and for any $u^{\prime} \in N_{G}(u) \cup N_{G}(v) \backslash\{u, v\}, u^{\prime} \in N_{G_{1}}(w)$. Thus

$$
\begin{aligned}
R^{\prime}\left(G_{1}\right)-R^{\prime}(G) & \sum_{u^{\prime} \in N_{G}(u) \backslash\{v\}}\left(\frac{1}{\max \left\{d_{G_{1}}(w), d_{G}\left(u^{\prime}\right)\right\}}-\frac{1}{\max \left\{d_{G}(u), d_{G}\left(u^{\prime}\right)\right\}}\right) \\
& +\sum_{u^{\prime} \in N_{G}(v) \backslash\{u\}}\left(\frac{1}{\max \left\{d_{G_{1}}(w), d_{G}\left(u^{\prime}\right)\right\}}-\frac{1}{\max \left\{d_{G}(v), d_{G}\left(u^{\prime}\right)\right\}}\right) \\
& +\frac{1}{d_{G_{1}}(w)}-\frac{1}{\max \left\{d_{G}(u), d_{G}(v)\right\}} \\
\leq & \frac{1}{d_{G_{1}}(w)}-\frac{1}{\max \left\{d_{G}(u), d_{G}(v)\right\}} \\
< & 0,
\end{aligned}
$$

as desired.

Lemma 9. Let $G \in \mathbb{B}_{6,3}$. Then $R^{\prime}(G) \geq 2$ with equality if and only if $G \cong B_{6,3}^{1}$ or $B_{6,3}^{2}$.

Proof. If $G$ has a pendent vertex with a neighbor of degree two, then $G$ is the graph obtained from the unique bicyclic graph on four vertices by attaching to a vertex (of degree two or three) a path on two vertices, and thus it is easily seen that $R^{\prime}(G)=\frac{13}{6}$ or $\frac{5}{2}$, implying that $R^{\prime}(G)>2$. Suppose that there is no pendent vertex 
with a neighbor of degree two. If there is no vertex of degree two in $G$, then $G$ is obtained by attaching a pendent vertex to every vertex of degree two of the unique bicyclic graph on four vertices, and thus $R^{\prime}(G)=\frac{7}{3}>2$. Suppose that there is a vertex, say $u$, of degree two in $G$. Let $v$ and $w$ be the two neighbors of $u$ in $G$. Then one of the two edges incident with $u$, say $u v \in M$, where $M$ is a perfect matching of $G$. Then $u$ lies on some cycle of $G$, and thus $G^{\prime}=G-u w$ is a unicyclic graph with a perfect matching. Let $a=d_{G}(w)$ and let $l$ be the number of neighbors of $w$ whose degree is at least $a$ in $G$. Note that $a \geq 2$. Then by Theorem $2, R^{\prime}(G)=$ $R^{\prime}\left(G^{\prime}\right)+\frac{1}{2} \geq \frac{3+1}{2}+\frac{1}{2}=\frac{5}{2}>2$ if $a=2$, and $R^{\prime}(G)=R^{\prime}\left(G^{\prime}\right)+\frac{a-l}{a}-\frac{a-l-1}{a-1}=$ $R^{\prime}\left(G^{\prime}\right)+\frac{l}{a(a-1)} \geq \frac{3+1}{2}=2$ with equality if and only if $G^{\prime} \cong U_{6,3}$ or $U_{6,3}^{*}$ and $l=0$, i.e., $G^{\prime} \cong U_{6,3}$ or $U_{6,3}^{*}$ and $w$ is a vertex of local maximal degree if $a \geq 3$. The result follows.

Lemma 10. [16] Let $G \in \mathbb{B}_{n, m}$ with $n>2 m \geq 6$, and $G$ has at least one pendent vertex. Then there is an m-matching $M$ and a pendent vertex $u$ such that $u$ is not $M$-saturated.

Let $g(m)=\frac{m+1}{2}-\frac{1}{12}$.

Let $\tilde{\mathbb{B}}(n)$ be the set of bicyclic graphs on $n$ vertices without pendent vertices, where $n \geq 4$. Let $\mathbb{B}_{n}^{1}(t)$ be the bicyclic graph with $n$ vertices containing two edge-disjoint cycles $C_{a}$ and $C_{b}$ that are joined by a path of length $t=n-a-b+1\left(C_{a}\right.$ and $C_{b}$ has a common vertex and $a+b=n+1$ if $t=0)$. Let $\mathbb{B}_{n}^{2}(s)$ be the set of bicyclic graphs obtained by joining two non-adjacent vertices of $C_{a}$ with $4 \leq a \leq n$ by a path of length $s=n-a+1$, where $n \geq 4$ and $s \geq 1$. Obviously, $\tilde{\mathbb{B}}(n)=\mathbb{B}_{n}^{1}(t) \cup \mathbb{B}_{n}^{2}(s)$.

Lemma 11. Let $G \in \mathbb{B}_{2 m, m}$ and no pendent vertex has neighbor of degree two, where $m \geq 4$. Then $R^{\prime}(G) \geq g(m)$ with equality if and only if $m=4$ and $G \cong B_{8,4}$.

Proof. Since $G \in \mathbb{B}_{2 m, m}$ and no pendent vertex has neighbor of degree two, $G$ is obtainable by attaching some pendent vertices to a graph in $\tilde{\mathbb{B}}(k)$, where $m \leq k \leq 2 m$, and any two pendent vertices have no common neighbor (if $k=2 m$, then no pendent vertex is attached).

Case 1. There is no vertex of degree two in $G$. Then either $k=m, G$ is obtainable by attaching a pendent vertex to every vertex of a graph $G_{1}$ in $\tilde{\mathbb{B}}(k)$, or $k=m+1$, $G$ is obtainable by attaching a pendent vertex to every vertex with degree two of a graph $G_{1} \in \mathbb{B}_{m+1}^{1}(1) \cup \mathbb{B}_{m+1}^{2}(1)$. By direct calculation, we have

$$
R^{\prime}(G)= \begin{cases}\frac{2}{3} m-\frac{1}{4} & \text { if } G_{1} \in \mathbb{B}_{m}^{1}(1) \cup \mathbb{B}_{m}^{2}(1), \\ \frac{2}{3} m-\frac{1}{3} & \text { if } G_{1} \in \mathbb{B}_{m}^{1}(t) \cup \mathbb{B}_{m}^{1}(0) \cup \mathbb{B}_{m}^{2}(t) \text { with } t \geq 2, \\ \frac{2}{3} m+\frac{1}{3} & \text { if } G_{1} \in \mathbb{B}_{m+1}^{1}(1) \cup \mathbb{B}_{m+1}^{2}(1) .\end{cases}
$$

Then $R^{\prime}(G) \geq g(m)$ with equality if and only if $m=4$ and $G \cong B_{8,4}$.

Case 2. There is a vertex, say $u$, of degree two in G. Denote by $v$ and $w$ the two neighbors of $u$ in $G$. Then one of the two edges incident with $u$, say $u v \in M$, where 
$M$ is a perfect matching of $G$. Suppose that there is no vertex of degree two in any cycle of $G$. Since no pendent vertex has neighbor of degree two in $G, u$ lies on the path joining the two disjoint cycles of $G$. For $G_{1}=G-u w+v w \in \mathbb{B}_{2 m, m}$, the difference of the numbers of vertices of degree two outside any cycle of $G$ and $G_{1}$ is equal to one, and thus by Lemma $8, R^{\prime}\left(G_{1}\right)<R^{\prime}(G)$. Repeating the operation from $G$ to $G_{1}$, we finally get a graph $G^{\prime} \in \mathbb{B}_{2 m, m}$, which has no vertex of degree two, such that $R^{\prime}(G)>R^{\prime}\left(G^{\prime}\right)$, and thus the result follows from Case 1. Now suppose that $u$ lies on some cycle of $G$. Consider $G^{\prime}=G-u w$, which is a unicyclic graph with perfect matching. Let $a=d_{G}(w)$ and let $k$ be the number of neighbors of $w$ whose degree is at least $a$ in $G$. Then by Theorem $2, R^{\prime}(G)=R^{\prime}\left(G^{\prime}\right)+\frac{1}{2} \geq \frac{m+1}{2}+\frac{1}{2}>$ $g(m)$ if $a=2$, and $R^{\prime}(G)=R^{\prime}\left(G^{\prime}\right)+\frac{k}{a(a-1)} \geq \frac{m+1}{2}>g(m)$ if $a \geq 3$.

Lemma 12. Let $G \in \mathbb{B}_{8,4}$. Then $R^{\prime}(G) \geq \frac{29}{12}$ with equality if and only if $G \cong B_{8,4}$.

Proof. If there is no pendent vertex with neighbor of degree two in $G$, then by Lemma $11, R^{\prime}(G) \geq g(4)=\frac{29}{12}$ with equality if and only if $G \cong B_{8,4}$. Suppose that $G$ has a pendent vertex $u$ whose neighbor $v$ is of degree two. Then $u v \in M$ and $G-u-v \in \mathbb{B}_{6,3}$. Let $w$ be the neighbor $v$ different from $u$. Let $a=d_{G}(w)$ and let $k$ the number of neighbors of $w$ whose degree is at least $a$. By Lemmas 2 and $9, R^{\prime}(G)=R^{\prime}(G-u-v)+1 \geq 2+1=3>\frac{29}{12}$ if $a=2$, and $R^{\prime}(G)=$ $R^{\prime}(G-u-v)+\frac{1}{2}+\frac{k}{a(a-1)} \geq 2+\frac{1}{2}+\frac{k}{a(a-1)} \geq \frac{5}{2}>\frac{29}{12}$ if $a \geq 3$.

Let $B_{n}(a, b)$ be the graph obtained from the unique bicyclic graph on four vertices by attaching to the two vertices of degree three $a-3$ and $b-3$ pendent vertices, respectively, where $a \geq b \geq 3, a+b=n+2$ and $n \geq 4$.

Theorem 3. Among the graphs in $\mathbb{B}_{n, 2}$ with $n \geq 6$,

$$
\begin{aligned}
R^{\prime}\left(B_{n}\left(\left\lceil\frac{n}{2}\right\rceil+1,\left\lfloor\frac{n}{2}\right\rfloor+1\right)\right) & >R^{\prime}\left(B_{n}\left(\left\lceil\frac{n}{2}\right\rceil+2,\left\lfloor\frac{n}{2}\right\rfloor\right)\right) \\
& >\cdots>R^{\prime}\left(B_{n}(n-1,3)\right)=\frac{5}{3},
\end{aligned}
$$

and for $G \in \mathbb{B}_{n, 2}$ not appearing in the above inequalities, $R^{\prime}(G)=2$.

Proof. Let $G \in \mathbb{B}_{n, 2}$. Then $G$ is of three types:

(1) $G=B_{n}(a, b)$ with $a \geq b \geq 3$. Then $R^{\prime}(G)=2-\frac{1}{b}$. It follows that

$$
\begin{aligned}
R^{\prime}\left(B_{n}\left(\left\lceil\frac{n}{2}\right\rceil+1,\left\lfloor\frac{n}{2}\right\rfloor+1\right)\right) & >R^{\prime}\left(B_{n}\left(\left\lceil\frac{n}{2}\right\rceil+2,\left\lfloor\frac{n}{2}\right\rfloor\right)\right) \\
& >\cdots>R^{\prime}\left(B_{n}(n-1,3)\right)=\frac{5}{3} .
\end{aligned}
$$

(2) $G$ is the graph obtained from the unique bicyclic graph on four vertices by attaching to a vertex of degree two $n-4$ pendent vertices . Then $R^{\prime}(G)=2$. 
(3) $G$ is the graph obtained from the unique bicyclic graph on five vertices in $\mathbb{B}_{5}^{2}(2)$ by attaching to one or two vertices of degree three some pendent vertices. Then $R^{\prime}(G)=2$.

Now the result follows easily.

Theorem 4. Let $G \in \mathbb{B}_{n, 3}$. Then $R^{\prime}(G) \geq 2$ with equality if and only if $G \cong B_{n, 3}^{1}$ or $G \cong B_{n, 3}^{2}$.

Proof. We prove the result by induction on $n$. If $n=6$, then the result follows from Lemma 9.

Suppose that $n \geq 7$ and the result holds for graphs in $\mathbb{B}_{n-1,3}$. Let $G \in \mathbb{B}_{n, 3}$. If there is no pendent vertex in $G$, then $G \in \tilde{\mathbb{B}}(n), n=7$, and thus

$$
\begin{aligned}
R^{\prime}(G) & = \begin{cases}3+\frac{1}{6} & \text { if } G \in \mathbb{B}_{7}^{1}(1) \cup \mathbb{B}_{7}^{2}(1), \\
3 & \text { otherwise, }\end{cases} \\
& >2
\end{aligned}
$$

Suppose that there is at least one pendent vertex in $G$. By Lemma 10, there is a 3-matching $M$ and a pendent vertex $u$ of $G$ such that $u$ is not $M$-saturated. Then $G-u \in \mathbb{B}_{n-1,3}$. Let $v$ be the unique neighbor of $u$. Let $a=d_{G}(v)$ and let $l$ be the number of neighbors of $v$ whose degree is at least $a$ in $G$. By Lemma 1, we have

$$
R^{\prime}(G)=R^{\prime}(G-u)+\frac{l}{a(a-1)} \geq 2+\frac{l}{a(a-1)} \geq 2
$$

with equalities if and only if $G-u \cong B_{n-1,3}^{1}$ or $B_{n-1,3}^{2}$ and $l=0$, i.e., $G-u \cong$ $B_{n-1,3}^{1}$ or $B_{n-1,3}^{2}$ and $v$ is a vertex of local maximal degree. Thus $R^{\prime}(G) \geq 2$ with equality if and only if $G \cong B_{n, 3}^{1}$ or $G \cong B_{n, 3}^{2}$

Theorem 5. Let $G \in \mathbb{B}_{n, m}$, where $n \geq 2 m$ and $m \geq 4$. Then $R^{\prime}(G) \geq g(m)$ with equality if and only if $G \cong B_{n, m}$.

Proof. We prove the result by induction on $n$ (for fixed $m$ ).

First suppose that $n=2 m$. We prove the result by induction on $m$.

If $m=4$, then from Lemma $12, R^{\prime}(G) \geq g(4)$ with equality if and only if $G \cong$ $B_{8,4}$.

Suppose that $m \geq 5$ and the result holds for graphs in $\mathbb{B}_{2 m-2, m-1}$. Let $G \in \mathbb{B}_{2 m, m}$ with a perfect matching $M$. If there is no pendent vertex with neighbor of degree two in $G$, then by Lemma $11, R^{\prime}(G)>g(m)$. Suppose that $G$ has a pendent vertex $u$ whose neighbor $v$ is of degree two. Then $u v \in M$ and $G-u-v \in \mathbb{B}_{2 m-2, m-1}$. Let $w$ be the neighbor of $v$ different from $u$. Let $a=d_{G}(w)$ and let $l$ be the number of neighbors of $w$ whose degree is at least $a$. By Lemma 2 and the induction hypothesis, $R^{\prime}(G)=R^{\prime}(G-u-v)+1 \geq g(m-1)+1>g(m)$ if $a=2$, and $R^{\prime}(G)=R^{\prime}(G-$ $u-v)+\frac{1}{2}+\frac{l}{a(a-1)} \geq g(m-1)+\frac{1}{2}+\frac{l}{a(a-1)} \geq g(m)$ with equalities if and only if $G-u-v \cong B_{2 m-2, m-1}$ and $l=0$, i.e., $G-u-v \cong B_{2 m-2, m-1}$ and $w$ is a vertex 
of local maximal degree if $a \geq 3$. Thus $R^{\prime}(G) \geq g(m)$ with equality if and only if $G \cong B_{2 m, m}$.

Suppose that $n>2 m$ and the result holds for graphs in $\mathbb{B}_{n-1, m}$. Let $G \in \mathbb{B}_{n, m}$. If there is no pendent vertex in $G$, then $G \in \tilde{\mathbb{B}}(2 m+1)$, and thus

$$
\begin{aligned}
R^{\prime}(G) & = \begin{cases}m+\frac{1}{6} & \text { if } G_{1} \in \mathbb{B}_{n}^{1}(1) \cup \mathbb{B}_{n}^{2}(1) \\
m & \text { otherwise }\end{cases} \\
& >g(m) .
\end{aligned}
$$

Suppose that there is at least one pendent vertex in $G$. By Lemma 10, there is an $m$-matching $M$ and a pendent vertex $u$ of $G$ such that $u$ is not $M$-saturated. Then $G-u \in \mathbb{B}_{n-1, m}$. Let $v$ be the unique neighbor of $u$. Let $a=d_{G}(v)$ and let $l$ be the number of neighbors of $v$ whose degree is at least $a$. By Lemma 1, we have

$$
R^{\prime}(G)=R^{\prime}(G-u)+\frac{l}{a(a-1)} \geq g(m)+\frac{l}{a(a-1)} \geq g(m)
$$

with equalities if and only if $G-u \cong B_{n-1, m}$ and $l=0$, i.e., $G-u \cong B_{n-1, m}$ and $v$ is a vertex of local maximal degree. Thus $R^{\prime}(G) \geq g(m)$ with equality if and only if $G \cong B_{n, m}$.

Corollary 5. Let $G$ be a bicyclic graph on $n \geq 6$ vertices. $R^{\prime}(G) \geq \frac{5}{3}$ with equality if and only if $G \cong B_{n}(n-1,3)$.

Proof. Let $m$ be the matching number of $G$. If $m \geq 4$, then by Theorem $5, R^{\prime}(G) \geq$ $g(m) \geq \frac{5}{2}-\frac{1}{12}>\frac{5}{3}$. If $m=3$, then by Theorem $4, R^{\prime}(G) \geq 2>\frac{5}{3}$. Thus the result follows from Theorem 3 .

Theorem 6. Let $G$ be a bicyclic graph on at least 5 vertices. Then $R^{\prime}(G) \leq \frac{n}{2}-\frac{1}{3}$ with equality if and only if $G \in \mathbb{B}_{n}^{1}(1) \cup \mathbb{B}_{n}^{2}(1)$.

Proof. Let $G$ be a graph with the maximum modified Randic index among the bicyclic graphs on $n$ vertices. By Lemmas 3 and $4, G \in \tilde{\mathbb{B}}(n)$. By direct calculation, we have

$$
R^{\prime}(G)= \begin{cases}\frac{n}{2}-\frac{1}{3} & \text { if } G \in \mathbb{B}_{n}^{1}(1) \cup \mathbb{B}_{n}^{2}(1), \\ \frac{n}{2}-\frac{1}{2} & \text { if } G \in \mathbb{B}_{n}^{1}(t) \cup \mathbb{B}_{n}^{2}(t) \cup \mathbb{B}_{n}^{1}(0) \text { with } t \geq 2 .\end{cases}
$$

The result follows.

\section{REFERENCES}

[1] V. Andova, M. Knor, P. Potočnik, and R. Škrekovski, "On a variation of Randić index," IMFM Preprint Series, vol. 49, no. 1156, 2011.

[2] B. Bollobás and P. Erdős, "Graphs of extremal weights," Ars Comb., vol. 50, pp. 225-233, 1998.

[3] M. Cygan, M. Pilipczuk, and R. Škrekovski, "On the inequality between radius and Randić index for graphs,” MATCH Commun. Math. Comput. Chem., vol. 67, pp. 451-466, 2012. 
[4] J. Devillers and A. T. Balaban, Eds., Topological indices and related descriptors in QSAR and QSPR. Amsterdam: Gordon and Breach, 1999.

[5] Z. Du and B. Zhou, "On sum-connectivity index of bicyclic graphs," Bull. Malays. Math. Sci. Soc. (2), vol. 35, no. 1, pp. 101-117, 2012.

[6] Z. Dvořák, B. Lidický, and R. Škrekovski, "Randić index and the diameter of a graph,” Eur. J. Comb., vol. 32, no. 3, pp. 434-442, 2011.

[7] I. Gutman and B. Furtula, Eds., Recent results in the theory of Randić Index. Kragujevac: Univ. Kragujevac, 2008.

[8] Y. Hou and J. Li, "Bounds on the largest eigenvalues of trees with a given size of matching," Linear Algebra Appl., vol. 342, no. 1-3, pp. 203-217, 2002.

[9] L. B. Kier and L. H. Hall, Molecular connectivity in structure-activity analysis. Wiley, 1986.

[10] X. Li and I. Gutman, Mathematical aspects of Randić-type molecular structure descriptors, ser. Mathematical Chemistry Monographs. Kragujevac: University of Kragujevac, Faculty of Science, 2005, vol. 1.

[11] L. Pogliani, "From molecular connectivity indices to semiempirical connectivity terms: Recent trends in graph theoretical descriptors," Chem. Rev., vol. 100, pp. 3827-3858, 2000.

[12] M. Randić, "On characterization of molecular branching," J. Amer. Chem. Soc., vol. 97, pp. 66096615,1975

[13] R. Todeschini and V. Consonni, Handbook of molecular descriptors. Weinheim: Wiley-VCH, 2000.

[14] A. Yu and F. Tian, "On the spectral radius of unicyclic graphs," MATCH Commun. Math. Comput. Chem., vol. 51, pp. 97-109, 2004.

[15] B. Zhou and N. Trinajstić, "On a novel connectivity index," J. Math. Chem., vol. 46, no. 4, pp. 1252-1270, 2009.

[16] Y. Zhu, G. Liu, and J. Wang, "On the Randić index of bicyclic conjugated molecules," in Recent results in the theory of Randić index. Kragujevac: Univ. Kragujevac, 2008, pp. 133-144.

\section{Authors' addresses}

\section{Jianping Li}

Faculty of Applied Mathematics, Guangdong University of Technology, Guangzhou 510090, P. R.

China, Department of Mathematics, South China Normal University, Guangzhou 510631, P. R. China

E-mail address: lijp06@163.com

\section{Bo Zhou}

Department of Mathematics, South China Normal University, Guangzhou 510631, P. R. China

E-mail address: zhoubodscnu.edu.cn 